\title{
A novel photochemical reaction of a mixed phosphonium iodonium ylide with phenylacetylene as a route to diphenyl- $1 \lambda^{5}$-phosphinoline
}

\author{
E. D. Matveeva, ${ }^{a \star}$ R. Gleiter, ${ }^{b}$ and N. S. Zefirov ${ }^{a}$ \\ ${ }^{a}$ Department of Chemistry, M. V. Lomonosov Moscow State University, \\ 1 Leninskie Gory, 119992 Moscow, Russian Federation. \\ Fax: +7 (495) 939 0290. E-mail: matveeva@org.chem.msu.ru \\ ${ }^{b}$ Institute of Organic Chemistry, Heidelberg University, Heidelberg, Germany. * \\ Fax: (0622) 154 4205. E-mail: Rolf.Gleiter@oci.uni-heidelberg.de
}

Earlier, we obtained mixed phosphonium iodonium ylides ${ }^{\mathbf{1}-\mathbf{4}}$ and studied their reactivities. In the present work, a novel photochemical reaction of ylide 1 with phenylacetylene afforded phenyl 1,1,4-triphenyl- $1 \lambda^{5}$-phosphinolin-2-yl ketone (2). The structure of $\lambda^{5}$-phosphinoline 2 was confirmed by NMR spectroscopy and elemental analysis.

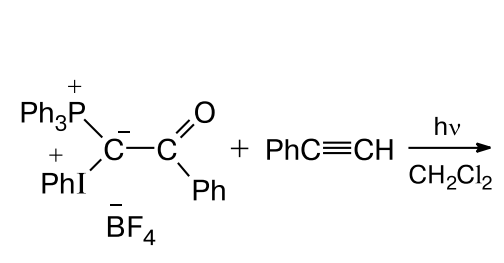

1

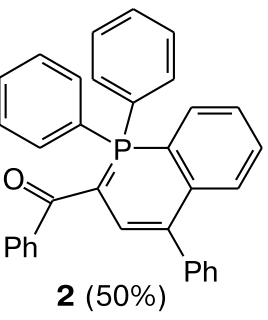

${ }^{1} \mathrm{H},{ }^{31} \mathrm{P}$, and ${ }^{13} \mathrm{C}$ NMR spectra were recorded on a Bruker Avance 400 instrument $(400 \mathrm{MHz})$ in $\mathrm{CD}_{2} \mathrm{Cl}_{2}$ and $\mathrm{CDCl}_{3}$ with tetramethylsilane as the internal standard. IR spectra were recorded on a UR-20 instrument in $\mathrm{CCl}_{4}$.

Phenyl 1,1,4-triphenyl-1 $\lambda^{5}$-phosphinolin-2-yl ketone (2). ylide $1(0.3 \mathrm{~g}, 0.45 \mathrm{mmol})$ in anhydrous $\mathrm{CH}_{2} \mathrm{Cl}_{2}$. The reaction mixture was stirred under argon, while being exposed to mercury lamp radiation. The completion of the reaction was indicated by the full consumption of ylide 1 (TLC). The reaction mixture was concentrated in vacuo. The residue was dissolved in a minimum amount of $\mathrm{CH}_{2} \mathrm{Cl}_{2}$ and separated by column chromatography on Merck 60 silica gel (70-230 mesh ASTM). Liberated PhI and the excess phenylacetylene were eluted with benzene and the target phosphinoline was eluted with $\mathrm{CH}_{2} \mathrm{Cl}_{2}$ and then $\mathrm{CH}_{2} \mathrm{Cl}_{2}-\mathrm{MeOH}(200: 1)$. The yield of compound 2 was $0.11 \mathrm{~g}(50 \%)$.

${ }^{1} \mathrm{H}$ NMR $\left(\mathrm{CD}_{2} \mathrm{Cl}_{2}\right), \delta: 7.12\left(\mathrm{~d}, 1 \mathrm{H}, \mathrm{CH},{ }^{3} \mathrm{~J}_{\mathrm{P}, \mathrm{H}}=31.5 \mathrm{~Hz}\right)$; 7.10-7.13 (m, 1 H, H arom.); 7.23-7.27 (m, 1 H, H arom.); 7.35-7.43 (m, $10 \mathrm{H}, \mathrm{H}$ arom.); 7.52-7.65 (m, $8 \mathrm{H}, \mathrm{H}$ arom.); (d, C(1), $\left.{ }^{1} J_{\mathrm{C}, \mathrm{P}}=100.5 \mathrm{~Hz}\right) ; 111.35\left(\mathrm{~d}, \mathrm{C}\right.$ arom., $\left.{ }^{1} J_{\mathrm{C}, \mathrm{P}}=85.8 \mathrm{~Hz}\right)$; Phenylacetylene $(0.1 \mathrm{~mL}, 0.9 \mathrm{mmol})$ was added to a solution of 7.84-7.90 (m, $4 \mathrm{H}, \mathrm{H}$ arom.). ${ }^{13} \mathrm{C} \mathrm{NMR}\left(\mathrm{CD}_{2} \mathrm{Cl}_{2}\right), \delta: 74.30$

$117.45\left(\mathrm{~d}, \mathrm{CHCPh},{ }^{3} J_{\mathrm{C}, \mathrm{P}}=10.0 \mathrm{~Hz}\right) ; 124.57$ (d, C arom., $\left.{ }^{3} J_{\mathrm{C}, \mathrm{P}}=11.5 \mathrm{~Hz}\right) ; 126.01\left(\mathrm{~d}, \mathrm{C}\right.$ arom. $\left.{ }^{2} J_{\mathrm{C}, \mathrm{P}}=7.6 \mathrm{~Hz}\right) ; 126.50$ (s, C arom.); 127.61 (d, C arom., ${ }^{1} J_{\mathrm{C}, \mathrm{P}}=93.5 \mathrm{~Hz}$ ); 128.46 (s, C arom.); 128.88 (s, C arom.); 129.14 (d, C arom., ${ }^{3} J_{\mathrm{C}, \mathrm{P}}=$ $=13.0 \mathrm{~Hz}) ; 129.24$ (s, C arom.); 130.00 (s, C arom.); 130.50 (s, C arom.); 132.05 (s, C arom.), 132.36 (d, C arom., ${ }^{4} J_{\mathrm{C}, \mathrm{P}}=$ $=3.1 \mathrm{~Hz}) ; 133.80\left(\mathrm{~d}, \mathrm{CH},{ }^{2} J_{\mathrm{C}, \mathrm{P}}=6.9 \mathrm{~Hz}\right) ; 133.89(\mathrm{~d}, \mathrm{C}$ arom., $\left.{ }^{2} J_{\mathrm{C}, \mathrm{P}}=10.7 \mathrm{~Hz}\right) ; 133.99\left(\mathrm{~d}, \mathrm{C}\right.$ arom., $\left.{ }^{3} J_{\mathrm{C}, \mathrm{P}}=9.2 \mathrm{~Hz}\right) ; 141.14$ (d, C arom., $\left.{ }^{3} J_{\mathrm{C}, \mathrm{P}}=9.9 \mathrm{~Hz}\right) ; 142.01\left(\mathrm{~d}, \mathrm{C}\right.$ arom.,$\left.{ }^{2} J_{\mathrm{C}, \mathrm{P}}=4.6 \mathrm{~Hz}\right)$; 143.32 (s, C arom.); 189.97 (d, CO, $\left.{ }^{2} J_{\mathrm{C}, \mathrm{P}}=6.1 \mathrm{~Hz}\right) .{ }^{31} \mathrm{P}$ NMR $\left(\mathrm{CDCl}_{3}\right), \delta: 2.94$. IR, v/cm $\mathrm{cm}^{-1}: 1580(\mathrm{C}=\mathrm{O}), 1520(\mathrm{C}=\mathrm{C})$, $720-750,1470$ (Ar). Found (\%): C, 85.24; H, 5.07. $\mathrm{C}_{34} \mathrm{H}_{25} \mathrm{OP}$. Calculated (\%): C, 85.00; H, 5.21.

We are grateful to A. S. Pavlova for performing the experimental work.

This work was financially supported by the Russian Foundation for Basic Research (Project No. 08-03-00611), the Presidium of the Russian Academy of Sciences (Basic Research Program No. 1), and the Division of Chemistry and Materials Science of the Russian Academy of Sciences (Basic Research Program No. 9).

\section{References}

1. E. D. Matveeva, T. A. Podrugina, Yu. K. Grishin, V. V. Tkachev, V. V. Zhdankin, S. M. Aldoshin, N. S. Zefirov, Zh. Org. Khim., 2003, 39, 572 [Russ. J. Org. Chem. (Engl. Transl.), 2003, 39].

2. E. D. Matveeva, T. A. Podrugina, Yu. K. Grishin, A. S. Pavlova, N. S. Zefirov, Zh. Org. Khim., 2007, 43, 209 [Russ. J. Org. Chem. (Engl. Transl.), 2007, 43].

3. E. D. Matveeva, T. A. Podrugina, A. S. Pavlova, A. V. Mironov, N. S. Zefirov, Izv. Akad. Nauk, Ser. Khim., 2008, 2195 [Russ. Chem. Bull., Int. Ed., 2008, 57, 2237].

4. E. D. Matveeva, T. A. Podrugina, A. S. Pavlova, A. V. Mironov, R. Gleiter, N. S. Zefirov, Eur. J. Org. Chem., 2009, 2323.

Received June 25, 2009,

* Organisch-chemisches Institut der Universität Heidelberg, Neuenheimer Feld 270 D-69120 Heidelberg, Deutschland. in revised form October 7, 2009 